\title{
A Case of Biliary Cystadenocarcinoma with Intracystic Bleeding
}

\author{
Aya Tani ${ }^{1}$, Hiroshi Yoshida', Yasuhiro Mamada ${ }^{1}$, \\ Nobuhiko Taniai ${ }^{1}$, Zenya Naito ${ }^{2}$ and Takashi Tajiri ${ }^{1}$ \\ ${ }^{1}$ Surgery for Organ Function and Biological Regulation, Graduate School of Medicine, Nippon Medical School \\ ${ }^{2}$ Department of Integrative Pathology, Graduate School of Medicine, Nippon Medical School
}

\begin{abstract}
Biliary cystadenocarcinomas are rare cystic tumors arising from the biliary epithelium. Symptoms vary and are sometimes absent. Most lesions are found incidentally during diagnostic radiologic procedures or surgical exploration for unrelated conditions. We describe an 80-year-old man with anemia due to intracystic bleeding. A computed tomographic scan showed a low-density hepatic lesion with thick, irregular walls and septa. Left lobectomy with cholecystectomy was performed. Histological examination revealed that the surgical specimen was a biliary cystadenocarcinoma. The concentrations of both carcinoembryonic antigen and CA19-9 were high in the cyst contents. The patient died of pneumonia 1 year 4 months after the operation, without the recurrence of cystadenocarcinoma.
\end{abstract}

(J Nippon Med Sch 2008; 75: 293-297)

Key words: hepatobiliary cystadenocarcinoma, surgery, intracystic bleeding

\section{Introduction}

Hepatobiliary

cystadenoma

and cystadenocarcinoma are rare cystic neoplasms of the liver. The wider use of imaging techniques, such as ultrasonography (US) and computed tomography (CT), has increased the number of reported cases. However, these neoplasms still represent less than $5 \%$ of all solitary nonparasitic cysts of biliary origin ${ }^{1}$. Despite the introduction of improved diagnostic techniques, the differential diagnosis of cystadenoma and cystadenocarcinoma, as well as of biliary cystadenomas and benign hepatic cysts, remains challenging. We describe a cystadenocarcinoma with intracystic bleeding that presented as anemia.

\section{Case Report}

The patient was an 80-year-old man with a 21year history of hypertension and senile dementia of Alzheimer type. He was admitted to our hospital because of anemia. On admission, a huge mass without tenderness was palpated in the upper abdomen. Laboratory studies on admission revealed the following: serum hemoglobin concentration, 8.7 $\mathrm{g} / \mathrm{dL}$ (normal, 14 to $18 \mathrm{~g} / \mathrm{dL}$ ); and serum gamma glutamic transpeptidase, $133 \mathrm{IU} / \mathrm{L}$ (normal, 8 to 39 $\mathrm{IU} / \mathrm{L})$. The serum levels of total bilirubin and $\alpha$ fetoprotein were within normal ranges. Hepatitis B antigen and hepatitis $\mathrm{C}$ antibody were negative.

No bleeding sites were detected on upper gastrointestinal endoscopy or colonoscopy.

Correspondence to Aya Tani, Department of Surgery, Nippon Medical School, 1-1-5 Sendagi, Bunkyo-ku, Tokyo 1138603, Japan

E-mail: ayatani@nms.ac.jp

Journal Website (http://www.nms.ac.jp/jnms/) 


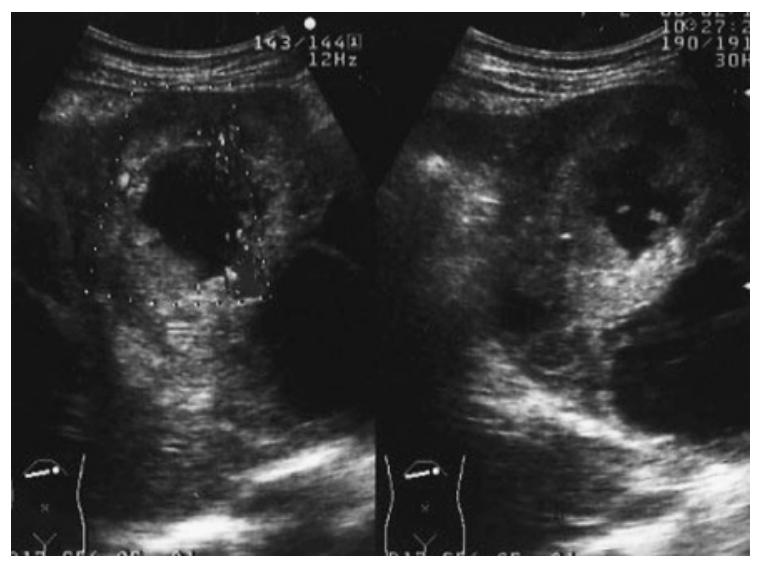

Fig. 1 Abdominal US revealed a unilocular oval mass with thick walls. The mass measured $10 \times 13 \mathrm{~cm}$ and was located in the left hepatic lobe.

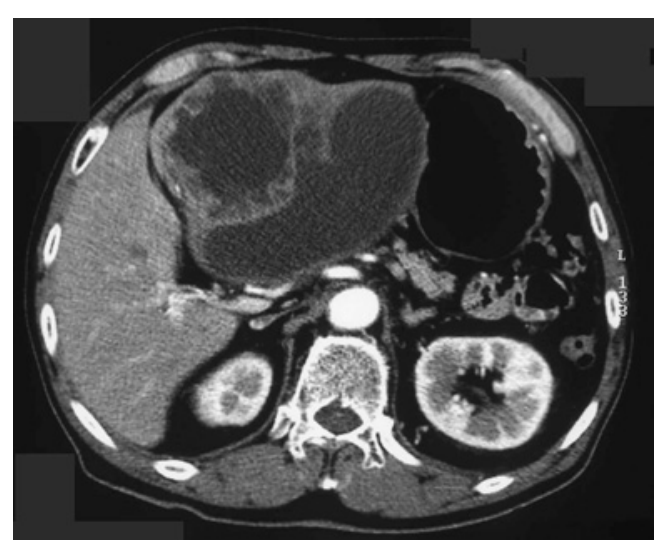

Fig. 2 A computed tomography showed a lowdensity hepatic lesion, measuring $12 \times 10$ $\mathrm{cm}$ in diameter, with thick, irregular walls and septa.

Abdominal US revealed a unilocular oval mass with thick walls. The mass measured $10 \times 13 \mathrm{~cm}$ and was located in the left hepatic lobe (Fig. 1). A CT scan showed a low-density hepatic lesion, measuring $12 \times$ $10 \mathrm{~cm}$ in diameter, with thick, irregular walls and septa ( Fig. 2) . Endoscopic retrograde cholangiography (ERC) showed no communication between the intrahepatic bile ducts and the cyst. On celiac angiography, the tumor appeared as an avascular lesion (Fig. 3a). The hepatic arteries were distended (Fig. 3a), and the portal vein was compressed; the left branch was occluded by the tumor (Fig. 3b). A T2-weighted magnetic resonance scan showed mural nodules with signal intensity higher than that of the liver (Fig. 4). Magnetic
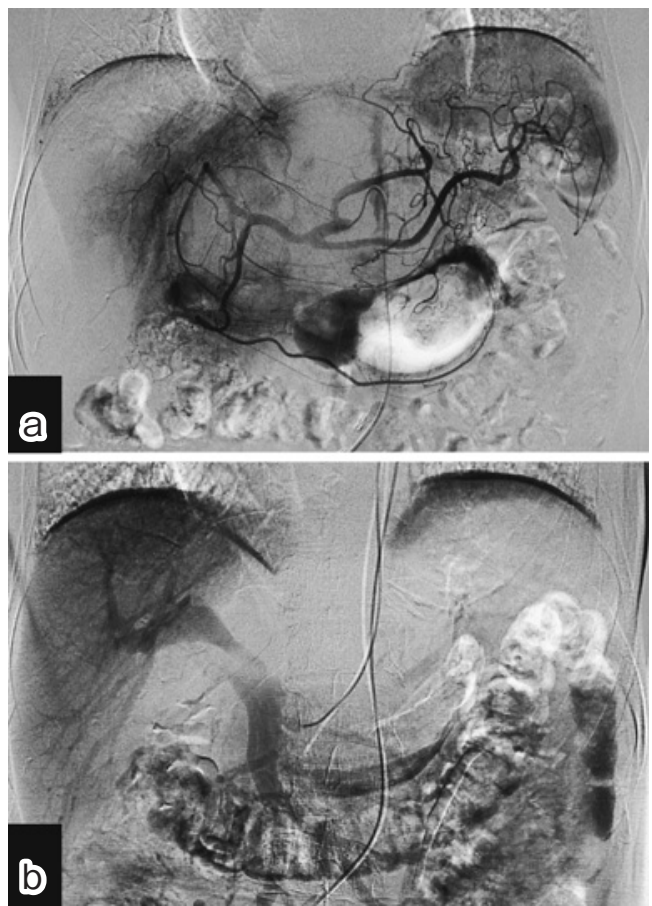

Fig. 3 The hepatic arteries were distended (a), and the portal vein was compressed; the left branch was occluded by the tumor (b).

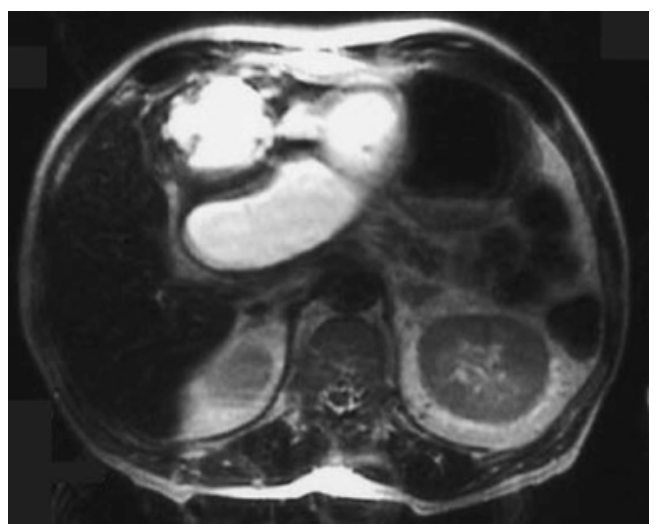

Fig. 4 A T2-weighted magnetic resonance image showed mural nodules with signal intensity higher than that of the liver.

resonance cholangiography showed no connection between the mass and the intrahepatic bile ducts. The cystic lesion was suspected of being a mucinproducing liver tumor, such as a cystadenoma or cystadenocarcinoma.

The patient underwent extensive left lobectomy with cholecystectomy. A large tumor with a smooth surface was present in the left lobe (Fig. 5a). The resected left lobe weighed 462 g. On macroscopic 

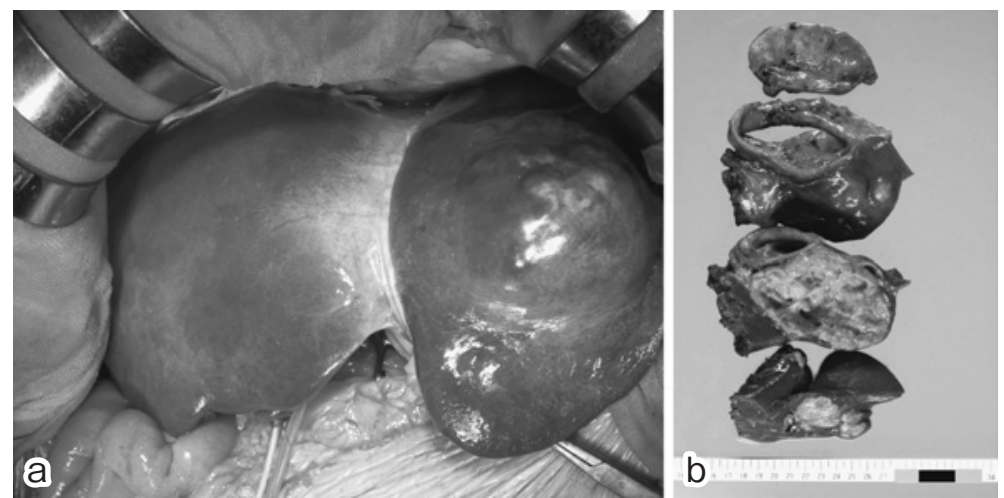

Fig. 5 A large tumor with a smooth surface was present in the left lobe (a). The resected left lobe weighed $462 \mathrm{~g}$. The resected multilocular tumor was filled with dark red fluid which appeared to be old blood, and the cut surface showed multiple, yellowish papillary nodules lining the cystic wall (b).
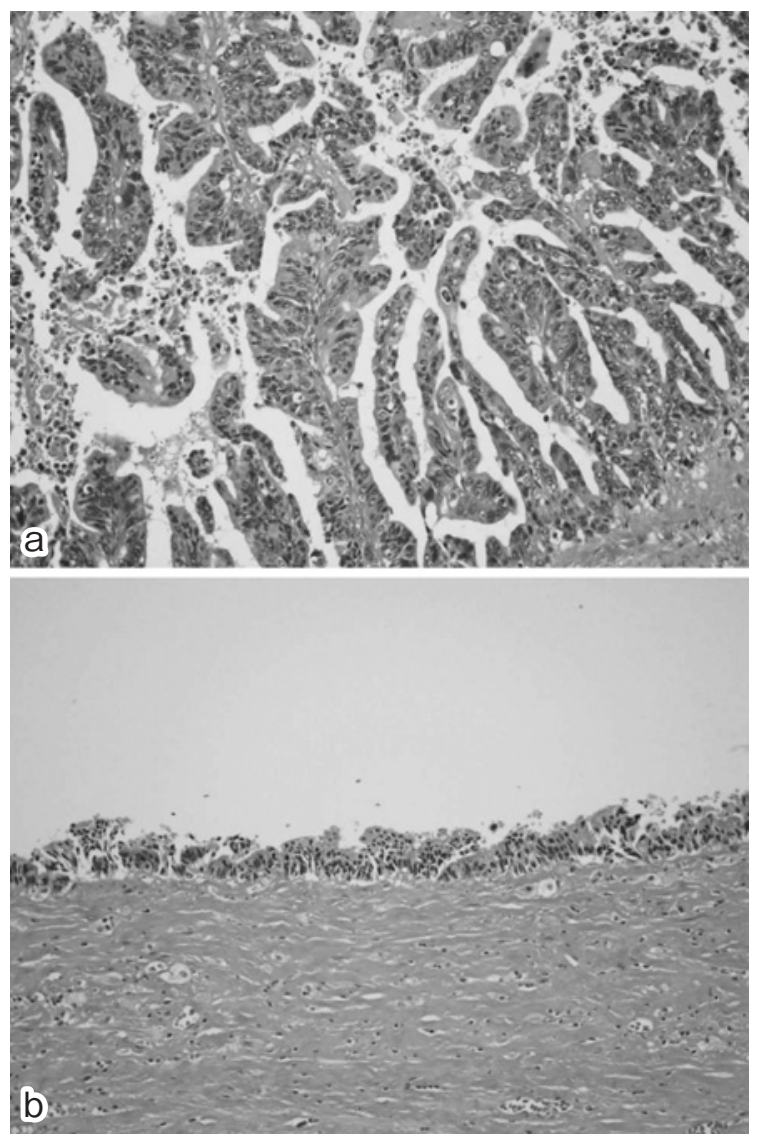

Fig. 6 On histologic examination, the tumor was a well-to-moderately differentiated adenocarcinoma with low papillary structures (a). The cyst wall consisted of a thick layer of hyalinized connective tissue without an ovarian-like stroma (b).

examination, the resected multilocular tumor was filled with a dark red fluid that appeared to be old blood, and the cut surface showed multiple, yellowish papillary nodules lining the cystic wall (Fig. 5b). On histologic examination, the tumor was a well-to-moderately differentiated adenocarcinoma with low papillary structures (Fig. 6a). The cyst wall consisted of a thick layer of hyalinized connective tissue without an ovarian-like stroma (Fig. 6b). Stromal invasion of the tumor was prominent. The concentrations of carcinoembronic antigen and CA19-9 in the cyst contents were 4,744 IU/L and $6,400,000 \mathrm{IU} / \mathrm{L}$, respectively. Massive invasion of the parenchyma of the left lobe was evident, but there was no metastasis to the lymph nodes in the hepatoduodenal ligament.

The patient's postoperative recovery was uneventful. Postoperatively, the maximum serum total bilirubin level was $0.6 \mathrm{mg} / \mathrm{dL}$. He died of pneumonia 16 months after operation without recurrence.

\section{Discussion}

Biliary cystadenocarcinomas are rare cystic tumors arising from the biliary epithelium. In 1943, Willis ${ }^{2}$ described the first case of hepatic cystadenocarcinoma. Although recent advances in diagnostic-imaging techniques, such as US, CT, and magnetic resonance, have facilitated the diagnosis of many abdominal tumors, biliary cystadenocarcinomas still account for only $0.41 \%$ of 
all malignant hepatic epithelial tumors ${ }^{3}$. As of 1998 only 112 cases have been reported in the literature ${ }^{4}$. Although our patient was an elderly man, most biliary cystadenocarcinomas occur in middle-aged women. Symptoms vary and include epigastric pain, a palpable abdominal mass, jaundice, and vomiting. Some patients are asymptomatic, with lesions being found incidentally during diagnostic radiologic procedures or surgical exploration for unrelated conditions. In our patient, the tumor was detected on a detailed evaluation of anemia due to intracystic bleeding.

Diagnostic-imaging techniques, such as CT and US, are useful for distinguishing simple cysts from biliary cystadenomas and cystadenocarcinomas, which have a more distinctive configuration. The presence of a solid, nodular, multilocular cystic mass with coarse calcifications along the walls or septa strongly suggests a cystadenocarcinoma. In our patient, the tumor appeared to be a cystadenocarcinoma.

It is often difficult to differentiate a cystadenocarcinoma from a cystadenoma on preoperative and intraoperative radiologic examinations. Although fine-needle aspiration and needle biopsy are useful diagnostic procedures, they should not be performed for differential diagnosis, because peritoneal carcinomatosis secondary to a biopsy of cystadenocarcinoma can occur ${ }^{6}$.

The pathogenesis of biliary cystadenocarcinoma is uncertain. Some authors propose a congenital origin from aberrant bile duct rests, whereas others favor an acquired etiology, such as a reactive process to some focal injury ${ }^{1}$. Woods ${ }^{7}$ have proposed that cystadenocarcinoma develops by malignant transformation of the epithelium of a benign cystadenoma. Crig and colleagues ${ }^{8}$ have reported the malignant transformation of cystadenomas in $25 \%$ of their patients. Further indirect support for malignant transformation is provided by a histochemical study showing that the expression of tumor-associated antigens, such as CA 19-9, carcinoembryonic antigen, early membrane antigen, and DUPAN-2, differs between cystadenoma and cystadenocarcinoma ${ }^{9}$.

Few molecular biologic studies of biliary cystadenocarcinomas have been performed. Genetic alterations similar to those found in intraductal papillary mucinous tumors of the pancreas are likely, occasionally associated with K-ras mutations in areas of low-grade and high-grade dysplasia ${ }^{10}$.

Devaney et $\mathrm{al}^{11}$. have divided cystadenocarcinomas into three subtypes, i.e., 1) cystadenocarcinoma with mesenchymal stroma arising from cystadenoma with mesenchymal stroma, occurring exclusively in females and following a relatively indolent course in most instances; 2) cystadenocarcinoma without mesenchymal stroma not associated with cystadenoma, occurring in males and following an extremely aggressive course ; and 3 ) cystadenocarcinoma without mesenchymal stroma, occurring in females and with a clinical course that is not well understood ${ }^{12,13}$.

Cystadenocarcinoma with mesenchymal stroma usually occurs in females and is believed to arise from cystadenoma with mesenchymal stroma, whereas cystadenocarcinoma without mesenchymal stroma is believed to arise from bile ducts or cholangiolar ducts, not from cystadenoma ${ }^{14}$. The present case may correspond to the second type of Devaney's classification, i.e., cystadenocarcinoma without mesenchymal stroma in a male.

The cystadenocarcinoma in our patient was detected on evaluation of anemia. Because no other bleeding sites were found on upper gastrointestinal or colonoscopic examinations, we attributed the anemia to intracystic bleeding. The treatment of choice is complete resection of the tumor with an adequate margin of normal liver tissue for both cystadenoma and cystadenocarcinoma, because reliable differentiation between these two lesions requires microscopic evaluation.

In conclusion, although hepatobiliary cystadenomas and cystadenocarcinomas are rare, these lesions should be included in the diagnostic diagnosis of patients with hepatic cysts. If hepatobiliary cystadenomas and cystadenocarcinomas cannot be excluded on the basis of radiologic findings, surgery is recommended. 


\section{References}

1. Ishak KG, Willis GW, Cummins SD, Bullock AA: Biliary cystadenoma and cystadenocarcinoma: report of 14 cases and review of the literature. Cancer 1977; 39: 322-338.

2. Willis RA: Carcinoma arising in congenital cysts of the liver. J Pathol Bacteriol 1943; 50: 492-495.

3. Takayasu K, Muramatsu Y, Moriyama N, et al.: Imaging diagnosis of bile duct cystadenocarcinoma Cancer 1988; 61: 941-946.

4. Läuffer JM, Baer HU, Maurer CA, Stoupis C, Zimmerman A, Búchler MW : Biliary cystadenocarcinoma of the liver: the need for complete resection. Eur J Cancer 1998; 34: 1845-1851.

5. Korobkin M, Stephens DH, Lee JK, et al.: Biliary cystadenoma and cystadenocarcinoma: $\mathrm{CT}$ and sonographic fidings. AJR Am J Roentgenol 1989; 153: 507-511.

6. Iemoto Y, Fukamachi S: Biliary cystadenocarcinoma with peritoneal carcinomatosis. Cancer 1981; 48: 1664-1667.

7. Woods GL: Biliary cystadenocarcinoma: case report of hepatic malignancy originating in benign cystadenoma. Cancer 1981; 47: 2936-2940.

8. Craig JR, Peters RL, Edomonson HA: Tumors of the liver and intrahepatic bile duct. In Atlas of tumoer pathology. Second series, fascicle 26, 1989; Armed
Forces Institute of Pathology, Washington DC.

9. Terada T, Nakamura Y, Ohta T, et al.: Mucinhistochemical and immunohistochemical profiles of epithelial cells of several types of hepatic cysts. Virchows Arch A Pathol Anat Histopathol 1991; 419: 499-504.

10. Yanagisawa A, Kato Y, Ohtake K, et al.: c-Ki-ras point mutations in ductectatic-type mucinous cystic neoplasms of the pancreas. Jpn J Cancer Rrs 1991; 82: $1057-1060$.

11. Devaney K, Goodman ZD, Ishak KG: Hepatobiliary cystadenoma and cystadenocarcinoma: a light microscopic and immunohistochemical study of 70 patients. Am J Surg Pathol 1994; 18: 1078-1091.

12. Akiyoshi T, Yamaguchi K, Chijiiwa K, Tanaka M: Cystadenocarcinoma of the liver without mesenchymal stroma: possible progression from a benign cystic lesion suspected by follow-up imagings. J Gastroenterol 2003; 38: 588-592.

13. Ishibashi Y, Ojima H, Hiraoka N, Sano T, Kosuge T, Kanai Y: Invasive biliary cystic tumor without ovarian-like stroma. Pathol Int 2007; 57: 794-798.

14. Akwari OE, Tucker A, Seigler HF, Itani KMF: Hepatobiliary cystadenoma with mesenchymal stroma. Ann Surg 1990; 211: 18-27.

(Received, February 27, 2008)

(Accepted, June 3, 2008) 